\title{
Hyperhomocysteinemia and MTHFR Polymorphisms as Antenatal Risk Factors of White Matter Abnormalities in Two Cohorts of Late Preterm and Full Term Newborns
}

\author{
Lucia M. Marseglia, ${ }^{1}$ Antonio Nicotera, ${ }^{2}$ Vincenzo Salpietro, ${ }^{3}$ Elisa Giaimo, ${ }^{2}$ \\ Giovanna Cardile, ${ }^{2}$ Maria Bonsignore, ${ }^{2}$ Angela Alibrandi, ${ }^{4}$ Daniela Caccamo, \\ Sara Manti, ${ }^{1}$ Gabriella D'Angelo, ${ }^{1}$ Carmelo Mami, ${ }^{6}$ and Gabriella Di Rosa ${ }^{2}$ \\ ${ }^{1}$ Department of Pediatric, Gynecological, Microbiological and Biomedical Sciences, Unit of Neonatal Intensive Care, \\ University of Messina, Messina, Italy \\ ${ }^{2}$ Department of Pediatric, Gynecological, Microbiological and Biomedical Sciences, Unit of Child Neurology and Psychiatry, \\ University of Messina, Messina, Italy \\ ${ }^{3}$ Department of Pediatric, Gynecological, Microbiological and Biomedical Sciences, Unit of Pediatric Genetics and Immunology, \\ University of Messina, Messina, Italy \\ ${ }^{4}$ Department of Economical, Business and Environmental Sciences and Quantitative Methods, University of Messina, Messina, Italy \\ ${ }^{5}$ Department of Biomedical Sciences and Morpho-Functional Imaging, University of Messina, Messina, Italy \\ ${ }^{6}$ Department of Pediatric, Gynecological, Microbiological and Biomedical Sciences, Unit of Neonatology, University of Messina, \\ Messina, Italy
}

Correspondence should be addressed to Gabriella Di Rosa; gdirosa@unime.it

Received 2 October 2014; Revised 29 January 2015; Accepted 29 January 2015

Academic Editor: Felipe Dal-Pizzol

Copyright (C) 2015 Lucia M. Marseglia et al. This is an open access article distributed under the Creative Commons Attribution License, which permits unrestricted use, distribution, and reproduction in any medium, provided the original work is properly cited.

Higher total homocysteine (tHcy) levels, and C677T and A1298C methylenetetrahydrofolate (MTHFR) polymorphisms, have been reported in preterm or full term newborns with neonatal encephalopathy following perinatal hypoxic-ischemic insult. This study investigated the causal role of tHcy and MTHFR polymorphisms together with other acquired risk factors on the occurrence of brain white matter abnormalities (WMA) detected by cranial ultrasound scans (cUS) in a population of late preterm and full term infants. A total of 171 newborns $(81 \mathrm{M}, 47.4 \%), 45$ (26.3\%) born $<37 \mathrm{wks}$, and $126(73.7 \%)$ born $\geq 37$ wks were recruited in the study. cUS detected predominant WMA pattern in 36/171 newborns (21.1\%) mainly characterized by abnormal periventricular white matter signal and mild-to-moderate periventricular white matter volume loss with ventricular dilatation $(6 / 36,16.6 \%)$. WMA resulted in being depending on tHcy levels $(P<0.014)$, lower GA $(P<0.000)$, lower Apgar score at 1 minutes $(P<0.000)$ and 5 minutes $(P<0.000)$, and 1298AC and 677CT/1298AC genotypes $(P<0.000$ and $P<0.000)$. In conclusion, both acquired and genetic predisposing antenatal factors were significantly associated with adverse neonatal outcome and WMA. The role of A1298C polymorphism may be taken into account for prenatal assessment and treatment counseling.

\section{Introduction}

Homocysteine (Hcy) is a sulfur-containing amino acid involved in methionine metabolism. Either genetic or environmental factors would influence total Hcy (tHcy) levels [1]. Cofactors deficiency or genetic polymorphisms of the key catabolic enzyme and methylenetetrahydrofolate reductase (MTHFR) may be the cause of increased levels of plasma homocysteine [1]. A direct correlation between higher tHcy levels and C677T and A1298C MTHFR polymorphisms has been reported in some populations of preterm or full term newborns with neonatal encephalopathy (NE) following 
perinatal hypoxic-ischemic (HI) insult [2]. However, the specific role of hyperhomocysteinemia and MTHFR polymorphisms in neonatal brain maturation and $\mathrm{HI}$ damage has not been unraveled. Mild hyperhomocysteinemia and inflammation have been associated with the development of several cerebrovascular diseases [3]. Mechanisms as excitotoxicity and activation of oxidative stress were related to the toxic effects of homocysteine [3]. Two definite patterns of brain injury were associated with NE following $\mathrm{HI}$ insult, predominantly involving white matter/watershed (WM/WS), or the basal ganglia/thalamus (BGT) regions $[2,4]$. The association between WM/WS pattern, tHcy levels, and either heterozygous or homozygous MTHFR C677T variants has been reported [2]. Nevertheless, beyond the mostly definite pattern of white matter injury such as periventricular leukomalacia (PVL), several qualitative neonatal white matter abnormalities (WMA) may account for different degrees of neurobehavioral impairment on long-term observation, mainly in very preterm $[5,6]$ but also in full term infants [4]. Both environmental events and predisposing genetic vulnerability were hypothesized as causative factors of WMA [7]. The aim of our study was to evaluate the role of tHcy and MTHFR polymorphisms together with other acquired risk factors on the occurrence of WMA detected by cranial ultrasound scans (cUS) in late preterm and full term infants.

\section{Subjects and Methods}

2.1. Clinical Data. Newborn infants were consecutively recruited at the Intensive Care Unit and Neonatology Unit of University Hospital of Messina between February 2011 and February 2013. Approval from the Local Ethical Committee was obtained for this study. Medical records were reviewed systematically for details concerning antenatal, perinatal, and neonatal course. Demographic and clinical data included sex, type of pregnancy (pathologic versus physiologic; singleton versus twin), drugs taken during pregnancy (steroids, tocolytics, progesterone, cardioaspirin or low-molecularweight heparin (LMWH)), mode of delivery (emergency cesarean section), preeclampsia, premature rupture of membranes (PROM), birth weight (BW; expressed in percentiles), gestational age (GA), Apgar score (1 and $5 \mathrm{~min}$ ), patent foramen ovale (PFO), perinatal adversities (bradycardia, altered flussimetry, seizures, infections, hypoglycemia, and multiorgan failure). Clinically recognizable neonatal encephalopathy after presumed HI was defined by abnormal tone pattern, feeding difficulties, altered alertness, or seizures, and at least one of the following criteria: (1) fetal distress at delivery, (2) requirement of resuscitation at birth, (3) arterial cord blood $\mathrm{pH}<7.1$, (4) 5-minute Apgar score $<7$, or (5) multiorgan failure [8]. Exclusion criteria included patients with GA below 32 wks, suspected or confirmed congenital brain malformations, inborn errors of metabolism, neuromuscular diseases, or trisomies.

2.2. Biochemical and Genetic Analyses. For measurement of tHcy levels in whole blood, samples were collected with $20 \mu \mathrm{L}$ of sodium heparinized capillaries (Bio-Rad Laboratories, Munich, Germany) within 1 week from birth. Then, blood samples were hemolyzed with $0.2 \mathrm{~mL}$ hemolysis reagent (aqueous solution of EDTA and a nonionic detergent; BioRad Laboratories), resulting in an immediate inhibition of Hcy-generating and -converting enzymes and a stabilization of the tHcy concentration for up to 2 days. The hemolyzed blood samples were stored at $+4^{\circ} \mathrm{C}$ for a maximum of 2 days until analysis. Measurements of plasma tHcy concentrations were performed by HPLC with kit dedicated (Bio-Rad Laboratories), according to manufacturer's instructions.

2.3. Genotyping of MTHFR Polymorphisms. The assessment of C677T and A1298C MTHFR genotypes was carried out on genomic DNA extracted from dried blood spots (DBS) samples. Written informed consent was obtained from patients' parents. DBS were obtained by heel prick with a sterile lancet, after applying filter paper to draining blood. Disks of $3 \mathrm{~mm}$ were cut out of the sampling paper and placed into $1.5 \mathrm{~mL}$ Eppendorf minitubes. Then, $500 \mu \mathrm{L}$ sterile water was added, and the minitubes were vortexed 3 times during 5 seconds. The water was pipetted off. After adding $200 \mu \mathrm{L}$ $10 \%$ Chelex-100 solution, the minitubes were placed in a water bath at $95^{\circ} \mathrm{C}$ for 30 minutes. Finally, these ready for use DNA solutions were pipetted into new minitubes. After DNA spectrophotometric quantification, genotyping for the C677T and A1298C polymorphisms was carried out by allelic discrimination technique in a 7500 real-time PCR instrument (Applied Biosystems), using Pre-Designed TaqMan_SNP Genotyping Assays (Applied Biosystems; assay ID: C_1202883_20 and C_850486_20, resp.), according to manufacturer's instructions. The frequency of C677T and A1298C MTHFR polymorphisms was compared between premature $(\mathrm{GA}<37)$ versus born-at-term newborns $(\mathrm{GA} \geq$ 37).

2.4. Neuroimaging Studies. Cerebral ultrasound was performed using an Esaote AUS with a $7.5 \mathrm{MHz}$ sector probe transducer to all newborns by the same experienced neonatologist. Serial cUSs were performed from 1 to 3 days of life and 44 postmenstrual age. Neonatal white matter abnormalities (WMA) were defined according to the presence of one of the following patterns: (1) white matter signal abnormalities, (2) periventricular white matter volume loss, (3) the presence of any cystic abnormalities, (4) ventricular dilatation, and (5) thinning of the corpus callosum [5].

2.5. Statistical Analyses. Numerical data were expressed as median and range and the categorical variables as numbers and percentages. Because numerical variables did not present normal distribution as verified by Kolmogorov Smirnov test and most of the measured variables were dichotomous or categorically ordered, nonparametric approach was used [9].

The Spearman correlation test was applied in order to assess the existence of interdependence between tHcy levels and other numerical parameters [9].

In order to assess the association between categorical variables (such as preterm versus full term newborns and MTHFR genotypes) we performed Chi-Square test. 
Odds ratio and related confidence interval at 95\% level were estimated in order to quantify the risk of premature birth for heterozygous MTHFR forms [9].

Mann-Whitney test was performed in order to compare tHcy levels between the groups of preterm versus full term infants and between WMA versus normal cUS scans. Kruskall-Wallis test was performed to compare tHcy levels among newborns with different Apgar scorings defined as normal (10-7), moderately impaired (6-4), and severely impaired (below 4) [9].

Finally, some logistic regression models [8] were estimated to verify the possible dependence of the WMA (in terms of Yes or NO) from GA, altered flussimetry, preeclampsia, PROM, bradycardia, tHcy levels, and MTHFR genotype. The same model was applied in order to assess the dependence of intraventricular hemorrhage (IVH) from the same potential explicative variables. Linear regression models were estimated in order to assess the dependence of numerical variables, such as BW, GA, and Apgar scores at $1 \mathrm{~min}$ and $5 \mathrm{~min}$ from tHcy levels. The same model was applied to ascertain the dependence of tHcy levels from drugs assumption during pregnancy (steroid, tocolytics, progestins, and cardioaspirin).

Statistical analyses were performed using SPSS 11.0 for Windows package. $P<0.05$, two-tailed, was considered to be statistically significant.

\section{Results}

3.1. Demographic and Clinical Features of the Sample. Summarized demographic, clinical, biochemical, and molecular features are shown in Table 1. A total of 171 newborns (81 M, 47.4\%), 45 (26.3\%) born <37 wks, and $126(73.7 \%)$ born $\geq 37$ wks were recruited. Pregnancy was physiologic in $104 / 171(60.8 \%)$, pathologic in $67 / 171(39.2 \%)$, single in $157 / 171(91.8 \%)$, and multiple in $14 / 171$ (8.2\%). Drugs' intake during gestation included steroid in 33/171 (19.3\%), tocolytics in $23 / 171$ (13.5\%), progesterone in $47 / 171$ (27.5\%), and cardioaspirin in $4 / 171(2.3 \%)$. Antenatal complications included preeclampsia in 5/171 (2.9\%) and PROM in $3 / 171$ (1.8\%). Perinatal complications included bradycardia in $18 / 171$ (10.5\%), altered flussimetry in 6/171 (3.5\%), and meconiumstained liquor in $3 / 171(1.7 \%)$. Neonatal data included the following: mean Apgar score $(1 \mathrm{~min})$ was $8.25, \mathrm{SD} \pm 1.7$ (median 9; range 0-10), mean Apgar score (5 min) was 9.35, $\mathrm{SD} \pm 1.1$ (median 10; range 3-10), mean BW percentile was 48.95, $\mathrm{SD} \pm 28.1$ (median 50; range 2-90), and mean GA was $37.2 \mathrm{SD} \pm 2.6$ (median 38; range $32-41$ ). Perinatal infections were diagnosed in 10/171 (5.8\%) of patients. Neonatal seizures occurred in 5/171 (2.9\%) of patients. Hypoglycemia $<2.0 \mathrm{mmol} / \mathrm{L}$ or multiorgan failure was evidenced in none of the patients.

3.2. Neuroimaging Findings. cUS showed that $36 / 171$ subjects (21.1\%) presented predominant WMA pattern mainly characterized by abnormal periventricular white matter signal and mild-to-moderate periventricular white matter volume loss with ventricular dilatation $(6 / 36,16.6 \%)$. Moreover, $2 / 171$ (1.1\%) showed BGT abnormalities, 13/171 (7.6\%) had intraventricular hemorrhage (IVH grade I-III), and 122/171 (71.3\%)
TABLE 1: Demographic, clinical, biochemical, neuroimaging and frequency features of the MTHFR genotypes. All genotypes were in Hardy-Weinberg equilibrium.

\begin{tabular}{|c|c|c|}
\hline Patients & \multicolumn{2}{|c|}{$N=171$} \\
\hline $\operatorname{Sex} N(\%)$ & \multicolumn{2}{|c|}{$81 \mathrm{M}(47.4 \%)$} \\
\hline \multicolumn{3}{|l|}{ Gestational age } \\
\hline$\geq 37$ & \multicolumn{2}{|c|}{$126(73.7 \%)$} \\
\hline$<37$ & \multicolumn{2}{|c|}{$45(26.3 \%)$} \\
\hline \multicolumn{3}{|l|}{ Type of pregnancy } \\
\hline Physiologic & \multicolumn{2}{|c|}{$104 / 171(60.8 \%)$} \\
\hline Pathologic & \multicolumn{2}{|c|}{$67 / 171(39.2 \%)$} \\
\hline Single & \multicolumn{2}{|c|}{ 157/171 (91.8\%) } \\
\hline Twin & \multicolumn{2}{|c|}{$14 / 171(8.2 \%)$} \\
\hline \multicolumn{3}{|l|}{ Drugs' intake during gestation } \\
\hline Steroid & \multicolumn{2}{|c|}{$33 / 171(19.3 \%)$} \\
\hline Tocolytics & \multicolumn{2}{|c|}{$23 / 171(13.5 \%)$} \\
\hline Progestins & \multicolumn{2}{|c|}{$47 / 171(27.5 \%)$} \\
\hline $\begin{array}{l}\text { Antiaggregant and/or } \\
\text { anticoagulant }\end{array}$ & \multicolumn{2}{|c|}{$4 / 171(2.3 \%)$} \\
\hline \multicolumn{3}{|l|}{ Antenatal complications } \\
\hline Preeclampsia & \multicolumn{2}{|c|}{$5 / 171(2.9 \%)$} \\
\hline PROM & \multicolumn{2}{|c|}{$3 / 171(1.8 \%)$} \\
\hline \multicolumn{3}{|l|}{ Perinatal complications } \\
\hline Fetal bradycardia & \multicolumn{2}{|c|}{$18 / 171(10.5 \%)$} \\
\hline Flussimetry & \multicolumn{2}{|c|}{$6 / 171(3.5 \%)$} \\
\hline Meconium-stained liquor & \multicolumn{2}{|c|}{$3 / 171(1.7 \%)$} \\
\hline \multicolumn{3}{|l|}{ Neonatal complications } \\
\hline Apgar score (1 min) & \multicolumn{2}{|c|}{$8.25(\mathrm{SD} \pm 1.7)$} \\
\hline Apgar score (5 min) & \multicolumn{2}{|c|}{$9.35(\mathrm{SD} \pm 1.1)$} \\
\hline Birth weight (percentile) & 48.9 & $\mathrm{SD} \pm 28.1)$ \\
\hline Perinatal infections & & $1(5.8 \%)$ \\
\hline Neonatal seizures & & $1(2.9 \%)$ \\
\hline Neuroimaging findings & & \\
\hline WMA & $36 /$ & $1(21.1 \%)$ \\
\hline Ventricular Dilatation & & $(16.6 \%)$ \\
\hline $\mathrm{BGT}$ & & $1(1.1 \%)$ \\
\hline IVH Grade I-II & & $1(7.6 \%)$ \\
\hline $\begin{array}{l}\text { Ventriculo-Peritoneal shunt } \\
\text { catheter }\end{array}$ & & $(46.1 \%)$ \\
\hline $\mathrm{tHcy} \mu \mathrm{M} / \mathrm{L}($ mean $\pm \mathrm{SD})$ & & \\
\hline$\geq 37 \mathrm{GA}$ & 9.74 & $\mathrm{D} \pm 3.98)$ \\
\hline$<37$ GA & 10.55 & $\mathrm{SD} \pm 4.29)$ \\
\hline Frequency of MTHFR C677T a & and $\mathrm{A} 1298 \mathrm{C} g$ & otypes $(N=77 \mathrm{pts})$ \\
\hline & MTHFR67 & 19CT (50\%) \\
\hline$\geq 37$ wks $(N=38)$ & & $4 \mathrm{TT}(10.5 \%)$ \\
\hline & MTHFR1298 & $6 \mathrm{AC}(15.7 \%)$ \\
\hline & & $1 C C(2.6 \%)$ \\
\hline & MTHFR677 & $25 \mathrm{CT}(64.1 \%)$ \\
\hline & & $4 \mathrm{TT}(10.2 \%)$ \\
\hline & MTHFR120 & $20 \mathrm{AC}(51.2 \%)$ \\
\hline & МАНГКО & $1 C C(2.5 \%)$ \\
\hline
\end{tabular}

GA: gestational age; tHcy: total homocysteine; MTHFR: methylenetetrahydrofolate reductase; SD: standard deviation; WMA: white matter abnormalities; BGT: basal ganglia/thalamus; IVH: intraventricular haemorrhage. 
showed unremarkable cUS scans. Ventricle-peritoneal shunt catheter was placed in 6 of the 13 patients $(46.1 \%)$ with IVH who developed hydrocephalus.

3.3. Biochemical Findings. No significant difference arose between mean tHcy values in premature infants (10.55 (SD $\pm 4.29) \mathrm{mM} / \mathrm{L}$, median $10.10 \mathrm{mM} / \mathrm{L}$ (range 5-26)) and full term infants $(9.74(\mathrm{SD} \pm 3.98) \mathrm{mM} / \mathrm{L}$, and median $9.55 \mathrm{mM} / \mathrm{L}$ (range 1.7-33)). Moreover, although, mean tHcy levels were higher in subjects with WMA pattern compared to those without cUS abnormalities, the difference only approached statistical significance $(P<0.081)$. Among the 171 subjects, $86(50.2 \%)$ showed tHcy values above the reference ranges for children age as for our laboratory (9 to $9.5 \mathrm{mM} / \mathrm{L}$ ) (Table 1 ).

3.4. Genetic Studies. Seventy-seven out of 171 subjects performed genetic analyses. The variants were distributed as CT (57.1\%) and TT (10.4\%) for C677T, AC (33.8\%) and CC (2.6\%) for A1298C. The genotype $677 \mathrm{~T} / 1298 \mathrm{C}$ was detected in 22 (28.6\%) subjects.

According to GA, MTHFR C677T polymorphisms were detected in 39/77 (50.6\%) preterm and in 38/77 (49.4\%) full term newborns, whereas MTHFR A1298C polymorphisms were evidenced in $21 / 39$ (53.8\%) preterms and in $7 / 38(18.4 \%)$ full terms. Within the group of preterm infants, 25/39 (64.1\%) bore 677CT genotype, 4/39 (10.2\%) bore 677TT genotype, $20 / 39(51.2 \%)$ bore 1298AC genotype, and 1/39 (2.5\%) bore $1298 \mathrm{CC}$ genotype. Within the group of full term infants, 19/38 (50\%) bore 677CT genotype, 4/38 (10.5\%) bore 677TT genotype, 6/38 (15.7\%) bore 1298AC genotype, and 1/38 $(2.6 \%)$ bore $1298 \mathrm{CC}$ genotype (Table 1$)$. Each genotype was in Hardy-Weinberg equilibrium.

3.5. Statistical Results. Significant correlations emerged between tHcy and PROM $(P<0.013)$, between tHcy and PFO $(P<0.042)$, and between tHcy levels and genotypes 1298AC $(P<0.000)$ and 677CT/1298AC $(P<0.000)$. In contrast, no significant correlations emerged between tHcy and sex, pregnancy type, steroids, tocolytics, progestins and cardioaspirin therapies, emergency cesarean section, preeclampsia, bradycardia, flussimetry, BW, GA, Apgar score at 1 and $5 \mathrm{mi}, \mathrm{IVH}$ and neonatal seizures. A strongly significant risk of premature birth was detected in association with MTHFR A1298C polymorphisms (OR = 5.61; 95\% CI $=1.91-16.44)$. In contrast, a moderate risk emerged between the MTHFR C677T polymorphisms and prematurity (OR $=1.78$ for CT; $95 \% \mathrm{CI}=0.71-4.44)$. Neonatal WMA pattern significantly covariated with MTHFR 677CT genotype $(P<0.041), 1298$ AC genotype $(P<0.000)$, and 677CT/ 1298AC $(P<0.000)$. Regression analysis showed that higher tHcy levels significantly influenced Apgar score at 5 min $(P<0.002)$ and occurrence of PROM $(P<0.007)$. Moreover, WMA resulted to be dependent on tHcy levels $(P<0.014)$, lower GA $(P<0.000)$, lower Apgar score at 1 minutes $(P<0.000)$ and 5 minutes $(P<0.000), 1298 \mathrm{AC}$, and $677 \mathrm{CT} / 1298 \mathrm{AC}$ genotypes $(P<0.000$ and $P<0.000)$. Statistical results are shown in Tables $2-4$.
TABle 2: $P$ values of Spearman correlation test between plasma tHcy levels and WMA with nonparametric variables.

\begin{tabular}{|c|c|}
\hline $\begin{array}{l}\text { Significance of covariation of the examined } \\
\text { variables and tHcy levels }\end{array}$ & $P$ values \\
\hline Sex $(M$ versus $F)$ & 0.223 \\
\hline Type of pregnancy & 0.225 \\
\hline Steroids & 0.718 \\
\hline Tocolytics & 0.332 \\
\hline Progestins & 0.240 \\
\hline Cardioaspirin & 0.084 \\
\hline Emergency cesarean section & 0.352 \\
\hline Preeclampsia & 0.413 \\
\hline PROM & $0.013^{*}$ \\
\hline Bradycardia & 0.636 \\
\hline Altered flussimetry & 0.664 \\
\hline Fetal distress & 0.444 \\
\hline BW & 0.531 \\
\hline GA & 0.670 \\
\hline Apgar $1 \mathrm{~min}$ & 0.446 \\
\hline Apgar $5 \mathrm{~min}$ & 0.298 \\
\hline WMA & 0.081 \\
\hline IVH & 0.864 \\
\hline Ventricular-peritoneal catheter & 0.454 \\
\hline $\mathrm{PFO}$ & $0.042^{*}$ \\
\hline Neonatal infections & 0.201 \\
\hline Neonatal seizures & 0.281 \\
\hline \multicolumn{2}{|l|}{ MTHFR genotypes } \\
\hline $677 \mathrm{CT}$ & 0.276 \\
\hline $677 \mathrm{TT}$ & 0.053 \\
\hline $1298 \mathrm{AC}$ & $0.000^{*}$ \\
\hline $1298 \mathrm{CC}$ & 0.232 \\
\hline $677 \mathrm{CT} / 1298 \mathrm{AC}$ & $0.000^{*}$ \\
\hline Nonparametric variables correlated to WMA & $P$ values \\
\hline $677 \mathrm{CT}$ & $0.041^{*}$ \\
\hline $677 \mathrm{TT}$ & 0.848 \\
\hline $1298 \mathrm{AC}$ & $0.000^{*}$ \\
\hline $677 \mathrm{CT} / 1298 \mathrm{AC}$ & $0.000^{*}$ \\
\hline
\end{tabular}

${ }^{*}$ Statistical significance. tHcy: total homocysteine; MTHFR: methylenetetrahydrofolate reductase; WMA: white matter abnormalities; IVH: intraventricular hemorrhage; PROM: premature rupture of membranes; BW: birth weight; PFO: patent foramen ovale; CT and TT: heterozygous and homozygous MTHFR 677 genotypes; AC and CC: heterozygous and homozygous MTHFR 1298 genotypes.

\section{Discussion}

The predisposing role of high tHcy levels and MTHFR polymorphisms on the occurrence of WMA has been strongly evidenced in our study, both in full-term and late preterm newborns. However, WMA was also significantly associated with acquired factors as GA and perinatal status, measured by Apgar scores. Several studies investigated on the origin and time of the neonatal brain lesions both in preterm and 
TABLE 3: Linear regression model showed dependence of Apgar score at 5 minutes, PFO, and PROM from tHcy levels.

\begin{tabular}{lc}
\hline tHcy & $P$ values \\
\hline Type of pregnancy & 0.958 \\
BW & 0.624 \\
GA & 0.374 \\
Apgar 1 & 0.065 \\
Apgar 5 & $\mathbf{0 . 0 0 2}$ \\
Steroids & 0.066 \\
Tocolytics & 0.545 \\
Progestins & 0.535 \\
Cardioaspirin & 0.167 \\
Preeclampsia & 0.447 \\
Emergency cesarean section & 0.704 \\
PROM & $\mathbf{0 . 0 0 7}$ \\
Bradycardia & 0.945 \\
PFO & $\mathbf{0 . 0 2 4}$ \\
Altered flussimetry & 0.469 \\
\hline
\end{tabular}

${ }^{*}$ Statistical significance, $P<0.05$. GA: gestational age; tHcy: total homocysteine; MTHFR: methylenetetrahydrofolate reductase; PROM: premature rupture of membranes; BW: birth weight; PFO: patent foramen ovale.

full term infants with NE [4, 10-13]. The role of intrapartum asphyxia in etiopathogenesis of NE has been debated and major neurological sequelae may not be evidenced in children who experienced fetal distress and perinatal asphyxia [14-16]. Mercuri et al. showed how 28\% of newborns presenting with NE with Apgar scores $<3$ had normal brain MRI scans or minor WMA, whereas 95\% of the infants with Apgar scores $>7$ and abnormal clinical assessment at 48 hours after birth had abnormal scans [11]. Cowan et al. reported that the pattern of brain injury depends on the duration and severity of the HI insult [10]. However, although the authors found that more than $90 \%$ of full term newborns with NE had a history of perinatal acquired insults, they could not exclude an additional role of other antenatal predisposing factors that make children more susceptible to the stressors during labour and delivery [10]. Moreover, the majority of patients with evidence of NE showed predominant localization of brain lesions in BGT and cortex regions, whereas the white matter regions appeared to be relatively spared within this group of children [10]. Notably, some authors reported that white matter damage is more seemingly related to long-standing antenatal risk factors interfering with brain development [4], and the state of brain maturation is an important prerequisite for this pattern of brain injury in full term newborns with $\mathrm{NE}$ $[4,17]$. The great contribution of lower GA at birth supported the hypothesis that development of WMA was critically related to maturation state of the brain [17]. The pathogenesis of WMA has been attributed to the intrinsic vulnerability of two cell types of the developing brain: preoligodendrocytes (pre-OLs) and subplate neurons [18, 19]. These cells result in being involved in critical developmental events between 24 and 40 weeks of GA [18], possibly disrupted by premature birth or intrauterine exposure to chronic hypoxic or inflammatory events $[17,20,21]$. Because of the rapidity
TABLE 4: Linear regression model showed dependence of WMA from tHcy levels, GA, Apgar scores at 1 and 5 minutes, 1298AC, and 677CT/1298AC MTHFR genotypes. IVH resulted in being dependent on GA and bradycardia by the same model.

\begin{tabular}{lc}
\hline WMA & $P$ values \\
\hline tHcy & $\mathbf{0 . 0 1 4 ^ { * }}$ \\
GA & $\mathbf{0 . 0 0 0 ^ { * }}$ \\
Apgar 1 & $\mathbf{0 . 0 0 0 ^ { * }}$ \\
Apgar 5 & $\mathbf{0 . 0 0 0 ^ { * }}$ \\
Altered flussimetry & 0.999 \\
Preeclampsia & 0.999 \\
PROM & 0.096 \\
677CT & 0.297 \\
677TT & 0.065 \\
1298AC & $\mathbf{0 . 0 0 0}$ \\
1298CC & 0.270 \\
677CT/1298AC & $\mathbf{0 . 0 0 0}$ \\
IVH & \\
tHcy & 0.862 \\
GA & $\mathbf{0 . 0 0 0 ^ { * }}$ \\
Preeclampsia & 0.314 \\
PROM & 0.138 \\
Altered flussimetry & 0.410 \\
Bradycardia & $\mathbf{0 . 0 0 0 *}$ \\
677CT & 0.726 \\
677TT & 0.522 \\
1298AC 98 CC & 0.101 \\
677CT/1298AC & 0.999 \\
\hline
\end{tabular}

${ }^{*}$ Statistical significance. $P<0.05$. tHcy: total homocysteine; MTHFR: methylenetetrahydrofolate reductase; WMA: white matter abnormalities; IVH: intraventricular hemorrhage; PROM: premature rupture of membranes; BW: birth weight; GA: gestational age; PFO: patent foramen ovale; CT and TT: heterozygous and homozygous MTHFR 677 genotypes; AC and CC: heterozygous and homozygous MTHFR 1298 genotypes.

and complexity of these changes, immature neural cells are deemed to be particularly vulnerable to various exogenous and endogenous insults, such as ischemia, inflammation, excitotoxicity, and free-radicals attack [18]. Determinants of the relative role of each scenario could be some factors such as GA, the timing and nature of the insult, additional risk factors such as disturbed nutritional state, drugs exposure, and many still-to-be-defined environmental or genetic parameters [18]. Within this complex context, Hcy might display its action by mediating oxidative stress activation, neurotoxicity, endothelial damage, or impaired blood coagulation [22]. A toxic role of Hcy on brain cortical and subcortical structures has also been reported to be mediated by the inhibition of $\mathrm{Na}+/ \mathrm{K}+$ ATPase. Several studies showed that chronic administration of Hcy to experimental animals induced a significant reduction of the activity of $\mathrm{Na}+\mathrm{K}+$ ATPase in cerebral cortex and hippocampus [23]. The critical role of this membrane protein on maintenance of ions homeostasis and subsequent neuronal excitability as well as transport and neurotransmitters uptake is well known [23]. Nevertheless, the activity 
of $\mathrm{Na}+\mathrm{K}+$ ATPase is known to be decreased by hypoxicischemic insults that, in central myelinated axons, lead to the accumulation of axoplasmic $\mathrm{Na}$ through noninactivating $\mathrm{Na}$ channels, which, together with membrane depolarization, promotes reverse $\mathrm{Na}-\mathrm{Ca}$ exchange and axonal $\mathrm{Ca}$ overload. Excessive accumulation of cytosolic $\mathrm{Ca}$ in turn activates various Ca-dependent enzymes resulting in irreversible injury [24]. Excitotoxic mechanisms induced by hypoxic-ischemic conditions also play an important role: glutamate released by reversal of $\mathrm{Na}$-dependent glutamate transporters activates AMPA/kainate receptors to cause injury to glia and myelin. Moreover, a toxic effect played by homocysteine has been reported in primary cultures of astrocytes as a result of impaired mitochondrial metabolism [25]. In the light of these considerations, higher tHcy levels might contribute to the neonatal WMA throughout several interplayed mechanisms at various molecular and cellular levels. Notably, we could only venture the induction of oxidative stress by higher tHcy because no specific oxidative parameters were determined in our newborns. de Kremer and Grosso first reported that antepartum high tHcy levels in mothers were significantly related to HI encephalopathy in their offspring [26]. Molloy et al. (2002) showed how plasma folate levels exert the highest influence on maternal tHcy, thus suggesting that lowering of maternal tHcy might be the key factor to prevent the subsequent risks in the fetus [27]. In contrast, Maayan-Metzger et al. recently reported increased tHcy levels positively related to BW, GA, multiparity, and MTHFR C677T but not A1298C polymorphisms. No association emerged between high tHcy levels and neonatal complications including those that can be attributed to vascular etiology such as PVL, necrotizing enterocolitis, and retinopathy [28]. Similarly Kenet et al. found no association in preterm infants between perinatal complications and thrombophilia [29]. Sturtz et al. reported on a trend for lower tHcy among infants with IVH but merely attributed these findings to nonfeeding of this very sick children group [30]. The first study investigating the subsequent relationship between thrombophilic risk factors and pattern of brain injury in full term infants with NE showed that the predominant impairment of WM/WS brain regions rather than $\mathrm{BGT}$ regions has been related to higher tHcy levels and MTHFR 677 CT or TT genotypes [2]. However, additional acquired factors such as emergency cesarean section, hypoglycemia $<2.0 \mathrm{mmol} / \mathrm{L}$, Apgar scores at 1 and $5 \mathrm{~min}$, umbilical artery $\mathrm{pH}$, and neonatal seizures played a significant causative role in this cohort [2]. Pogliani et al. showed that no significant differences were observed between the mothers, homozygous for MTHFR C677T, among their newborns with or without evidence of cUS abnormalities, with regards to age, ethnicity, type of delivery, tHcy, genetic thrombophilic factors, and drugs during pregnancy. There were no significant differences in gestational age, birth weight, umbilical cord $\mathrm{pH}$, Apgar scores, or smallfor-gestational age babies [31]. Abnormal cUS scans were detected in spite of maternal folic acid supplementation and antithrombotic prophylaxis during pregnancy [31]. The same authors showed a high incidence of cerebral abnormalities in neonates born to women with C677T homozygous mutation in the MTHFR gene detected by cUS [32].
The causal relationship between MTHFR A1298C polymorphisms on premature birth and neonatal WMA was less reported than MTHFR C677T variant. Moreover, studies on the role of MTHFR A1298C polymorphisms and pediatric cerebrovascular disorders gave conflicting results. MTHFR A1298C variant has been associated with ischemic stroke mainly in Asian but not in Caucasian populations $[33,34]$. However, other studies did not demonstrate this correlation [35]. Two female cousins with neonatal stroke, one with compound heterozygous A1298C and C677T MTHFR variants and the other with heterozygous A1298C polymorphism, were reported [36]. An increased risk of small-for-gestational age (SGA) outcome was associated with maternal C677T and A1298C MTHFR polymorphisms in a population of sixtysix women who gave birth to one or more SGA babies [37]. Cizmeci et al. first reported a case of cerebral sinovenous thrombosis associated with MTHFR A1298C mutation in the neonatal period [38]. Conversely, homozygosity for MTHFR 1298 resulted in being protective against severe preeclampsia, fetal growth restriction, placental abruption, stillbirth, or neonatal death in a wide population of healthy nulliparous women [39]. In conclusion, both acquired (GA and perinatal events) or genetic predisposing antenatal factors were significantly associated with adverse neonatal outcome and WMA in our population of newborns, both involving activation of neurotoxicity and oxidative stress. Possibly, newborns with antenatal thrombophilic risk factors might request specific perinatal assessment, treatment, and care. Finally, the role of A1298C polymorphism may be taken into account for prenatal assessment and treatment counseling.

\section{Conflict of Interests}

The authors declare that there is no conflict of interests regarding the publication of this paper.

\section{References}

[1] A. Maayan-Metzger, A. Lubetsky, J. Kuint et al., "The impact of genetic and environmental factors on homocysteine levels in preterm neonates," Pediatric Blood \& Cancer, vol. 60, no. 4, pp. 659-662, 2013.

[2] J. C. Harteman, F. Groenendaal, M. J. Benders, A. Huisman, H. J. Blom, and L. S. de Vries, "Role of thrombophilic factors in full-term infants with neonatal encephalopathy," Developmental Medicine and Child Neurology, vol. 55, no. 7, pp. 642-653, 2013.

[3] E. B. S. Scherer, S. O. Loureiro, F. C. Vuaden et al., "Mild hyperhomocysteinemia increases brain acetylcholinesterase and proinflammatory cytokine levels in different tissues," Molecular Neurobiology, vol. 50, no. 2, pp. 589-596, 2014.

[4] A. M. Li, V. Chau, K. J. Poskitt et al., "White matter injury in term newborns with neonatal encephalopathy," Journal of Pediatrics, vol. 147, no. 5, pp. 609-616, 2005.

[5] A. J. Spittle, J. Cheong, L. W. Doyle et al., "Neonatal white matter abnormality predicts childhood motor impairment in very preterm children," Developmental Medicine and Child Neurology, vol. 53, no. 11, pp. 1000-1006, 2011.

[6] S. E. G. Hamrick, S. P. Miller, C. Leonard et al., "Trends in severe brain injury and neurodevelopmental outcome in premature newborn infants: the role of cystic periventricular 
leukomalacia," Journal of Pediatrics, vol. 145, no. 5, pp. 593-599, 2004.

[7] S. Calkavur, M. Akisu, O. Olukman et al., "Genetic factors that influence short-term neurodevelopmental outcome in term hypoxic-ischaemic encephalopathic neonates," The Journal of International Medical Research, vol. 39, no. 5, pp. 1744-1756, 2011.

[8] J. J. Volpe, "Hypoxic-ischemic encephalopathy: clinical aspects," in Neurology of the Newborn, pp. 331-196, W.B. Saunders, Philadelphia, Pa, USA, 4th edition, 2000.

[9] D. G. Kleimbaum and M. Klein, Logistic Regression. A SelfLearning Text, Springer, New York, NY, USA, 2nd edition, 2002.

[10] F. Cowan, M. Rutherford, F. Groenendaal et al., "Origin and timing of brain lesions in term infants with neonatal encephalopathy," The Lancet, vol. 361, no. 9359, pp. 736-742, 2003.

[11] E. Mercuri, M. Rutherford, A. Barnett et al., "MRI lesions and infants with neonatal encephalopathy. Is the Apgar score predictive?” Neuropediatrics, vol. 33, no. 3, pp. 150-156, 2002.

[12] A. Leviton and O. Dammann, "Coagulation, inflammation, and the risk of neonatal white matter damage," Pediatric Research, vol. 55, no. 4, pp. 541-545, 2004.

[13] S. P. Miller, D. M. Ferriero, C. Leonard et al., "Early brain injury in premature newborns detected with magnetic resonance imaging is associated with adverse early neurodevelopmental outcome," Seminars Pediatric Neurology, vol. 16, no. 4, pp. 167178, 2009.

[14] K. B. Nelson and J. K. Grether, "Potentially asphyxiating conditions and spastic cerebral palsy in infants of normal birth weight," American Journal of Obstetrics \& Gynecology, vol. 179, no. 2, pp. 507-513, 1998.

[15] N. Badawi, J. J. Kurinczuk, J. M. Keogh et al., "Intrapartum risk factors for newborn encephalopathy: the Western Australian case-control study," British Medical Journal, vol. 317, no. 7172, pp. 1554-1558, 1998.

[16] N. Badawi, J. J. Kurinczuk, J. M. Keogh et al., "Antepartum risk factors for newborn encephalopathy: the Western Australian case-control study," British Medical Journal, vol. 317, no. 7172, pp. 1549-1553, 1998.

[17] S. A. Back and S. P. Miller, "Brain injury in premature neonates: a primary cerebral dysmaturation disorder?" Annals of Neurology, vol. 75, no. 4, pp. 469-486, 2014.

[18] J. J. Volpe, "The encephalopathy of prematurity-brain injury and impaired brain development inextricably intertwined," Pediatric Research, vol. 73, no. 1, pp. 80-86, 2013.

[19] J. J. Volpe, "Brain injury in premature infants: a complex amalgam of destructive and developmental disturbances," The Lancet Neurology, vol. 8, no. 1, pp. 110-124, 2009.

[20] O. Baud, J.-L. Daire, Y. Dalmaz et al., "Gestational hypoxia induces white matter damage in neonatal rats: a new model of periventricular leukomalacia," Brain Pathology, vol. 14, no. 1, pp. 1-10, 2004.

[21] P. Svedin, I. Kjellmer, A.-K. Welin, S. Blad, and C. Mallard, "Maturational effects of lipopolysaccharide on white-matter injury in fetal sheep," Journal of Child Neurology, vol. 20, no. 12, pp. 960-964, 2005.

[22] A. M. Troen, "The central nervous system in animal models of hyperhomocysteinemia," Journal of Child Neurology, vol. 22, no. 6, pp. 753-755, 2007.

[23] E. B. S. Scherer, S. O. Loureiro, F. C. Vuaden et al., "Mild hyperhomocysteinemia reduces the activity and immunocontent, but does not alter the gene expression, of catalytic $\alpha$ subunits of cerebral $\mathrm{Na}^{+}, \mathrm{K}^{+}$-ATPase," Molecular and Cellular Biochemistry, vol. 378, no. 1-2, pp. 91-97, 2013.

[24] E. L. Streck, C. Matte, P. S. Vieira et al., "Reduction of $\mathrm{Na}^{+}, \mathrm{K}^{+}$ATPase activity in hippocampus of rats subjected to chemically induced hyperhomocysteinemia," Neurochemical Research, vol. 27, no. 12, pp. 1593-1598, 2002.

[25] Y. Jin and L. Brennan, "Effects of homocysteine on metabolic pathways in cultured astrocytes," Neurochemistry International, vol. 52, no. 8, pp. 1410-1415, 2008.

[26] R. D. de Kremer and C. Grosso, "Maternal mutation 677C > T in the methylenetetrathydrofolate reductase gene associated with severe brain injury in offspring," Clinical Genetics, vol. 67, no. 1, pp. 69-80, 2005.

[27] A. M. Molloy, J. L. Mills, J. McPartlin, P. N. Kirke, J. M. Scott, and S. Daly, "Maternal and fetal plasma homocysteine concentrations at birth: the influence of folate, vitamin $B_{12}$, and the 5,10-methylenetetrahydrofolate reductase $677 \mathrm{C} \rightarrow \mathrm{T}$ variant," American Journal of Obstetrics and Gynecology, vol. 186, no. 3, pp. 499-503, 2002.

[28] A. Maayan-Metzger, A. Lubetsky, J. Kuint et al., "The impact of genetic and environmental factors on homocysteine levels in preterm neonates," Pediatric Blood \& Cancer, vol. 60, no. 4, pp. 659-662, 2013.

[29] G. Kenet, A. Maayan-Metzger, N. Rosenberg et al., “Thrombophilia does not increase risk for neonatal complications in preterm infants," Thrombosis and Haemostasis, vol. 90, no. 5, pp. 823-828, 2003.

[30] W. J. Sturtz, K. H. Leef, A. B. Mackley, S. Sharma, T. Bottiglieri, and D. A. Paul, "Homocysteine levels in preterm infants: is there an association with intraventricular hemorrhage? a prospective cohort study," BMC Pediatrics, vol. 7, article 38, 2007.

[31] L. Pogliani, L. Muggiasca, L. Arrigoni, E. Rossi, and G. Zuccotti, "Maternal methylenetetrahydrofolate reductase (MTHFR) homozygosity and neonatal outcome: follow-up of 42 pregnancies at risk," Journal of Child Neurology, vol. 25, no. 6, pp. 701-704, 2010.

[32] L. Pogliani, C. Cerini, F. Penagini, P. Duca, C. Mameli, and G. V. Zuccotti, "Cerebral ultrasound abnormalities in offspring of women with C677T homozygous mutation in the MTHFR gene: a prospective study," World Journal of Pediatrics, 2014.

[33] N. K. Kim and B. O. Choi, "MTHFR A1298C gene polymorphism: independent risk factor for ischemic stroke?" Journal of the Korean Neurological Association, vol. 21, pp. 606-613, 2003.

[34] Q. Q. Lv, J. Lu, W. Wu, H. Sun, and J. S. Zhang, "Association of the methylenetetrahydrofolate reductase gene A1298C polymorphism with stroke risk based on a meta-analysis," Genetics and Molecular Research, vol. 12, no. 4, pp. 6882-6894, 2013.

[35] S. Kang, Y. Wu, L. Liu, X. Zhao, and D. Zhang, "Association of the A1298C polymorphism in MTHFR gene with ischemic stroke," Journal of Clinical Neuroscience, vol. 21, no. 2, pp. 198202, 2014.

[36] M. R. Golomb, M. Heiny, and B. P. Garg, "Two cousins with neonatal stroke, PAI-1 4G variant and MTHFR A1298C mutation," Journal of Child Neurology, vol. 22, no. 6, pp. 753-755, 2007.

[37] N. Özbeka, F. B. Ataçb, H. Verdib, S. Çetintaşa, B. Gürakan, and A. Haberald, "Relationship between small-for-gestational age births and maternal thrombophilic mutations," Thrombosis Research, vol. 122, no. 2, pp. 175-178, 2008. 
[38] M. N. Cizmeci, M. K. Kanburoglu, A. Z. Akelma et al., “Cerebral sinovenous thrombosis associated with MTHFR A1298C mutation in the newborn: a case report," Journal of Thrombosis and Thrombolysis, vol. 35, no. 2, pp. 279-281, 2013.

[39] J. M. Said, J. R. Higgins, E. K. Moses et al., "Inherited thrombophilia polymorphisms and pregnancy outcomes in nulliparous women," Obstetrics and Gynecology, vol. 115, no. 1, pp. 5-13, 2010. 


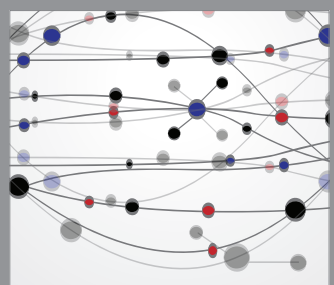

The Scientific World Journal
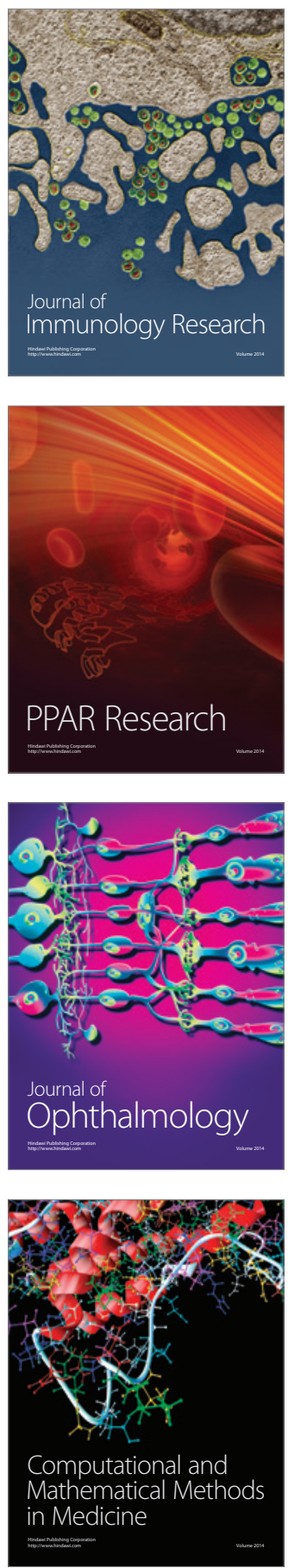

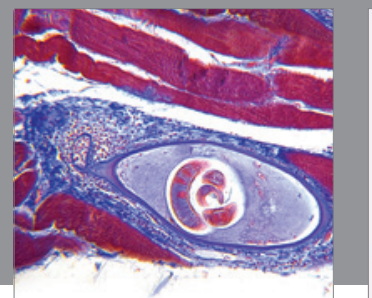

Gastroenterology

Research and Practice
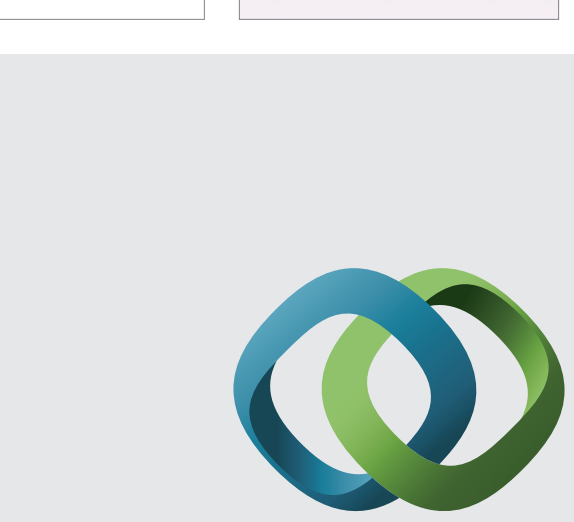

\section{Hindawi}

Submit your manuscripts at

http://www.hindawi.com
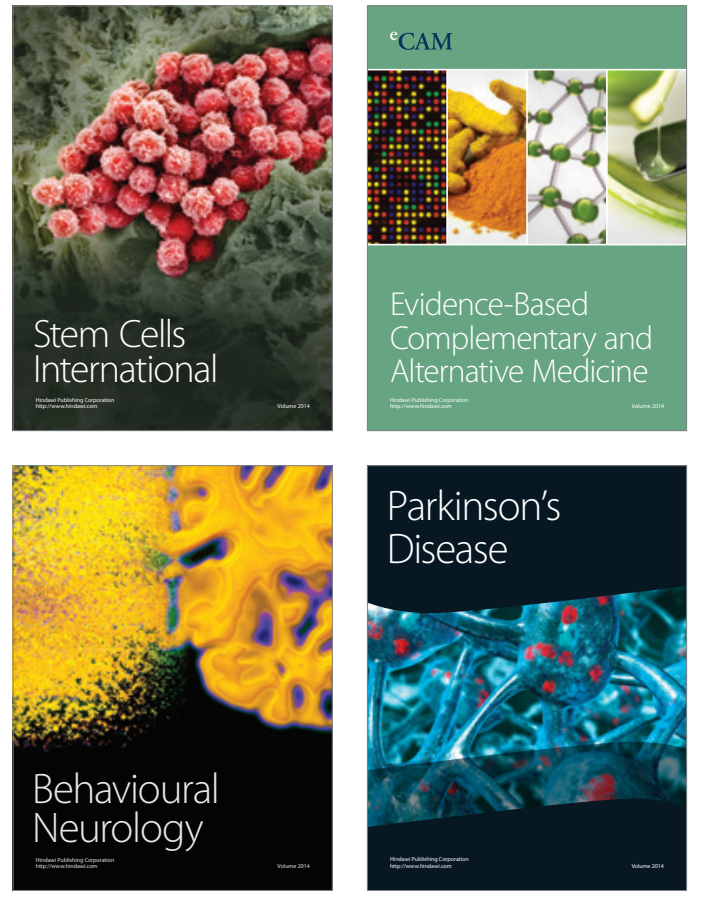
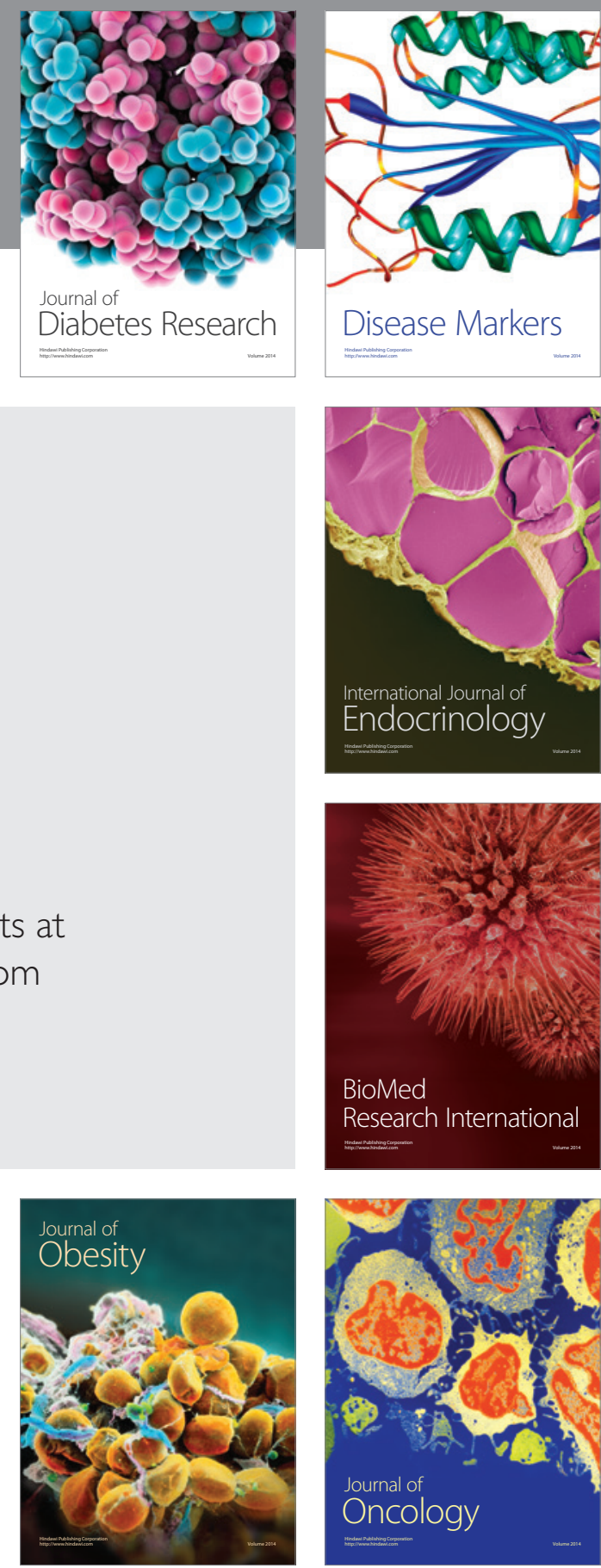

Disease Markers
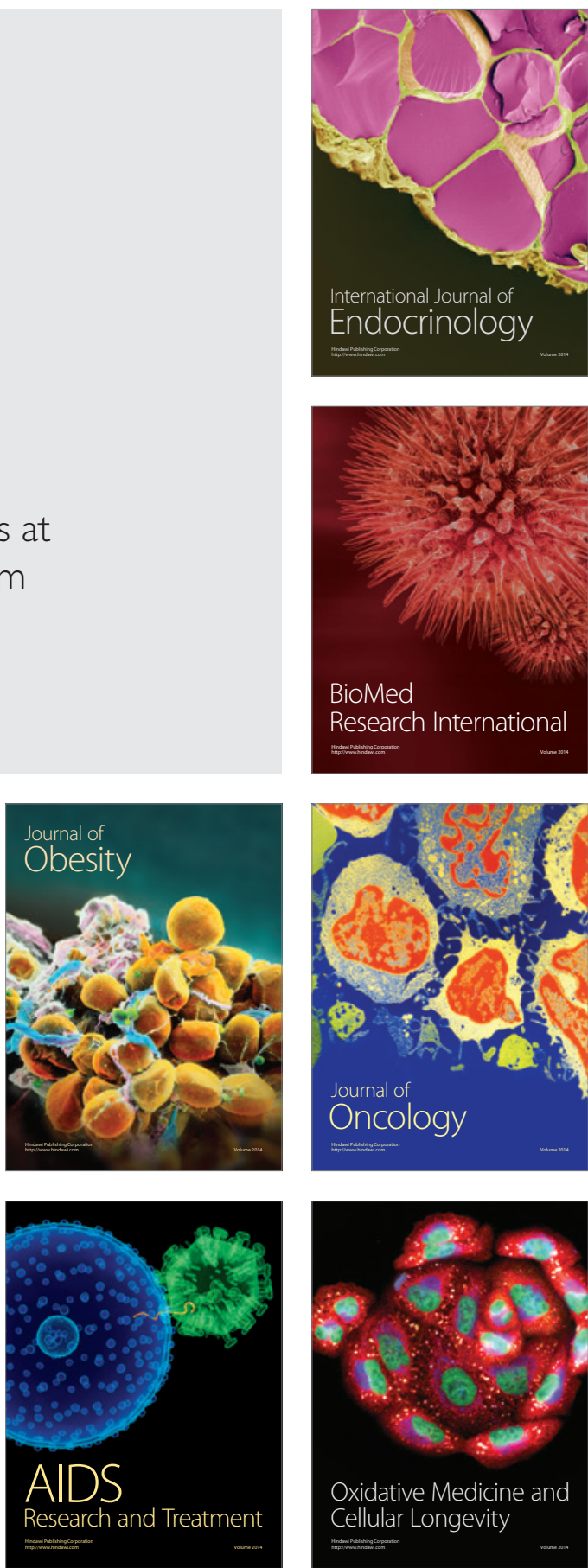